\title{
Reversible Left Ventricular Wall Thickening with Takotsubo Syndrome Sequentially Detected by Cardiac Magnetic Resonance Imaging
}

\author{
Hideyuki Hayashi ${ }^{1}$, Masahiro Kimura ${ }^{2}$, Takao Kato ${ }^{2}$, Kazutaka Nakasone ${ }^{1}$, Yuta Seko ${ }^{1}$, \\ Takayuki Sekihara ${ }^{1}$, Yuki Kimura ${ }^{1}$, Moritoshi Funasako ${ }^{1}$, Kenichi Sasaki ${ }^{1}$, Eisaku Nakane ${ }^{1}$, \\ Shoichi Miyamoto ${ }^{1}$, Toshiaki Izumi ${ }^{1}$, Tetsuya Haruna ${ }^{1}$ and Moriaki Inoko ${ }^{1}$
}

\begin{abstract}
:
Recently, it has been reported that spontaneous left ventricular wall thickening occurs among patients with takotsubo syndrome, which affects the long-term prognosis of such patients due to cerebral and cardiac complications. We herein report two cases of transient left ventricular wall thickening with takotsubo syndrome in which sequential cardiac magnetic resonance imaging revealed the existence of edematous changes in the thickened wall. Notably, a left ventricular aneurysm was detected during the course of ventricular wall thickening and may have played a role in the development of serious complications accompanied by takotsubo syndrome. This is the first case report of left ventricular aneurysm occurring with ventricular wall thickening due to takotsubo syndrome.
\end{abstract}

Key words: ventricular aneurysm, cardiac MRI, myocardial edema, takotsubo cardiomyopathy, hypertrophy, takotsubo syndrome

(Intern Med 57: 517-522, 2018)

(DOI: 10.2169/internalmedicine.9065-17)

\section{Introduction}

Functional recovery after takotsubo syndrome occurs within one to two weeks. Spontaneous left ventricular wall thickening (LVWT) after the recovery of the cardiac function has recently been reported $(1,2)$. While it has been reported that transient LVWT affects the long-term prognosis (3), transient LVWT in patients with takotsubo syndrome is not uncommon and is often associated with cardiac complications. Inflammation with hemodynamic instability, such as septic shock, is a precipitating factors of transient LVWT (3). However, the mechanism by which LVWT develops and its effects on the clinical course of patients have not yet been fully investigated.

We herein report two cases of transient LVWT with takotsubo syndrome in which sequential magnetic resonance im- aging (MRI), in part, illustrated these mechanisms.

\section{Case Reports}

\section{Case 1}

Case 1 was a 90-year-old woman who presented with aspiration pneumonia without chest pain. Her medical history included aortic valve replacement because of symptomatic severe aortic stenosis. Upon admission, she was hemodynamically stable, with a $\mathrm{SpO}_{2}$ value of $98 \%\left(\mathrm{O}_{2} 1 \mathrm{~L} / \mathrm{min}\right)$, but auscultation revealed coarse crackles in the bilateral lung fields. She exhibited an elevated troponin I level $(0.21 \mathrm{ng} /$ $\mathrm{mL})$ and decreased left ventricular apical wall motion [ejection fraction (EF), 26\%], both of which had not been seen in her before (Fig. 1A and B). Her 12-lead electrocardiogram findings showed $\mathrm{T}$ wave inversion in leads I, II, III,

${ }^{1}$ Cardiovascular Center, The Tazuke Kofukai Medical Research Institute Kitano Hospital, Japan and ${ }^{2}$ Department of Cardiovascular Medicine, Kyoto University Graduate School of Medicine, Japan

Received: February 20, 2017; Accepted: July 5, 2017; Advance Publication by J-STAGE: December 8, 2017

Correspondence to Dr. Masahiro Kimura, mkimura@kuhp.kyoto-u.ac.jp 

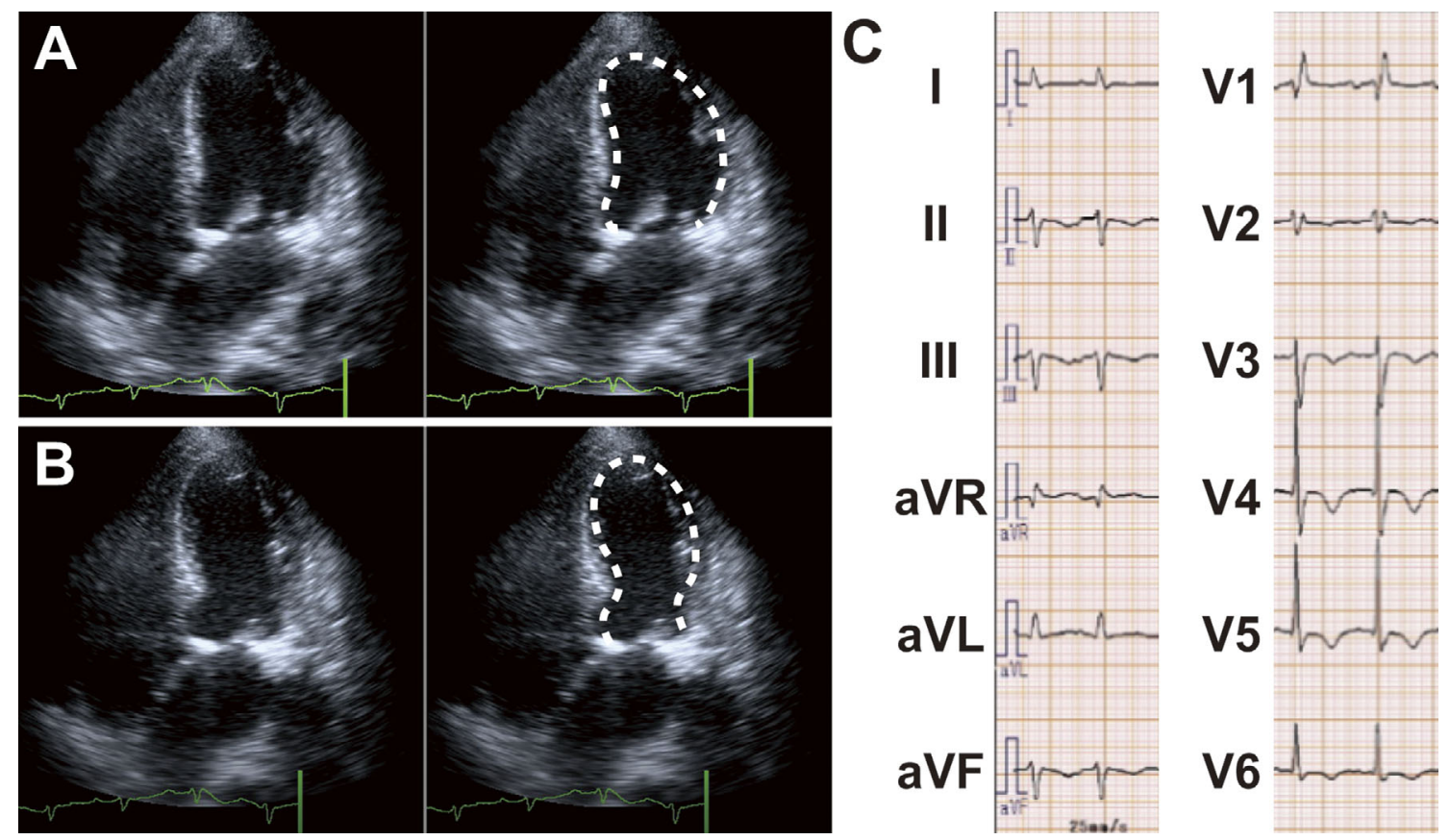

Figure 1. Echocardiography images and electrocardiography from case 1 at the onset of takotsubo syndrome. Apical wall motion abnormality was detected by echocardiography in end-diastole (A) and end-systole (B). Her electrocardiogram showed $T$ wave inversion in multiple leads (C).

aVF, and V3-V6 (Fig. 1C). Coronary computer tomography indicated no obstructive coronary artery disease or acute plaque rupture. Therefore, a diagnosis of takotsubo syndrome was made.

One month after the onset, her 12-lead electrocardiogram showed a flattening of $\mathrm{T}$ waves in leads I, II, III, aVF, and V3-V6. LVWT developed following improvement in the impaired contraction, and a left ventricular aneurysm that had never been observed in previous studies was detected by echocardiography (Fig. 2). This change was more remarkably observed using cardiac MRI than echocardiography (Fig. 3A-D). The T2-weighted signal intensity at the apex was greater than at the base (Fig. 3I). The relative myocardial T2 signal intensity (SI) ratio (4), calculated as $\mathrm{SI}_{\text {myocardium }} /$ $\mathrm{SI}_{\text {skeletalmuscle, was }} 2.22$, indicating that marked myocardial edema occurred after the impaired contraction recovered. Focal late gadolinium enhancement (LGE) was detected in the apex.

Four months after the onset, she was followed-up by echocardiography and cardiac MRI. The LVWT had diminished, and the T2 SI ratio decreased to 1.76 , but the left ventricular aneurysm persisted (Fig. 3E-H and J). Focal LGE in the apex was unclear because of thinning of the aneurysmal wall. The $\mathrm{T}$ wave changes in the 12-lead electrocardiogram recovered.

\section{Case 2}

Case 2 was a 78-year-old woman who presented with acute onset chest pain that was triggered by acute emotional stress. Her medical history included well-controlled hypertension, hypertensive nephropathy, and colon carcinoma treated with surgery. She did not have coronary artery disease, such as angina. At admission, she was hemodynamically stable and had no dyspnea. Her 12-lead electrocardiogram exhibited $\mathrm{T}$ wave inversions in leads I, aVL, and V1-V 6 (Fig. 4A). She exhibited an elevated troponin I level (3.89 $\mathrm{ng} / \mathrm{mL}$ ) and decreased left ventricular apical wall motion (EF $42 \%$ ), neither of which had been seen in her before. Emergent coronary angiography was performed, and the angiogram showed an absence of obstructive coronary artery disease. However, left ventriculography showed apical akinesia, basal hyperkinesia, and left ventricular apical ballooning (Fig. 4B and C), which prompted us to make a diagnosis of takotsubo syndrome.

One month after onset, LVWT occurred after the impaired contraction improved (Fig. 5A-D). Her 12-lead electrocardiogram exhibited giant negative $\mathrm{T}$ waves in leads I, II, III, aVL, aVF, and V1-V6. Four months after the onset, the LVWT had diminished (Fig. 5E-H), and the relative myocardial T2 SI ratio recovered from 4.67 (Fig. 5I, 1 month after the onset) to 1.87 (Fig. 5J). Her 12-lead electrocardiogram indicated that she had recovered from the $\mathrm{T}$ wave inversion. In this case, no left ventricular aneurysm was detected using echocardiography or cardiac MRI. These changes were more easily assessed using MRI than echocardiography. Gadolinium-based imaging was avoided because of severe kidney disease (estimated glomerular filtration rate: $22 \mathrm{~mL} /$ $\min / 1.73 \mathrm{~m}^{2}$ ). 

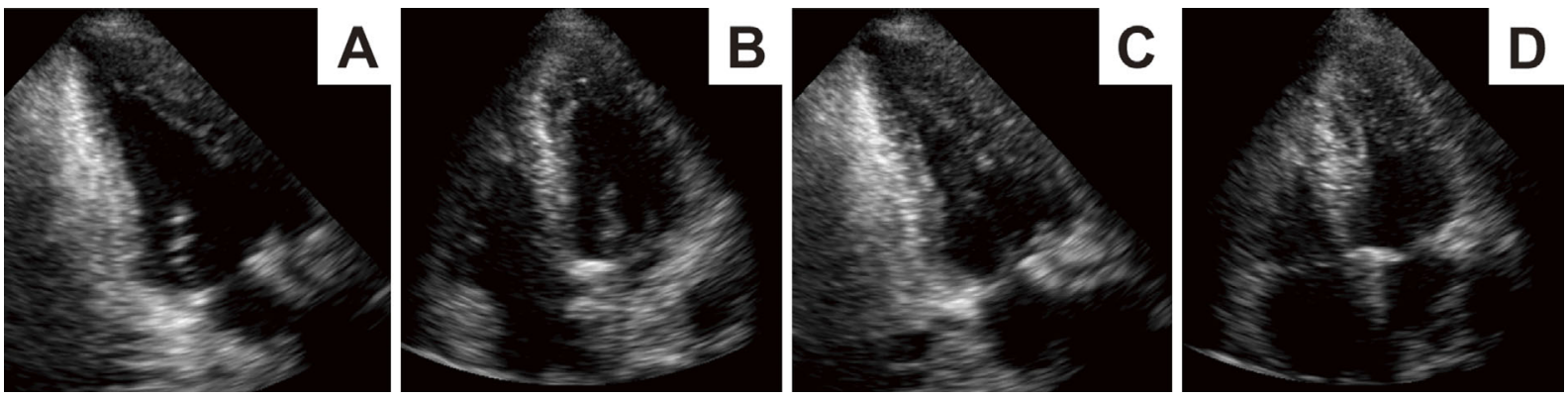

Figure 2. Echocardiography images from case 1 at 18 days after the onset of takotsubo syndrome. LVWT and left ventricular aneurysm were detected using apical four-chamber and two-chamber views in end diastolic (A and B) and end systolic frames (C and D). LVWT: left ventricular wall thickening
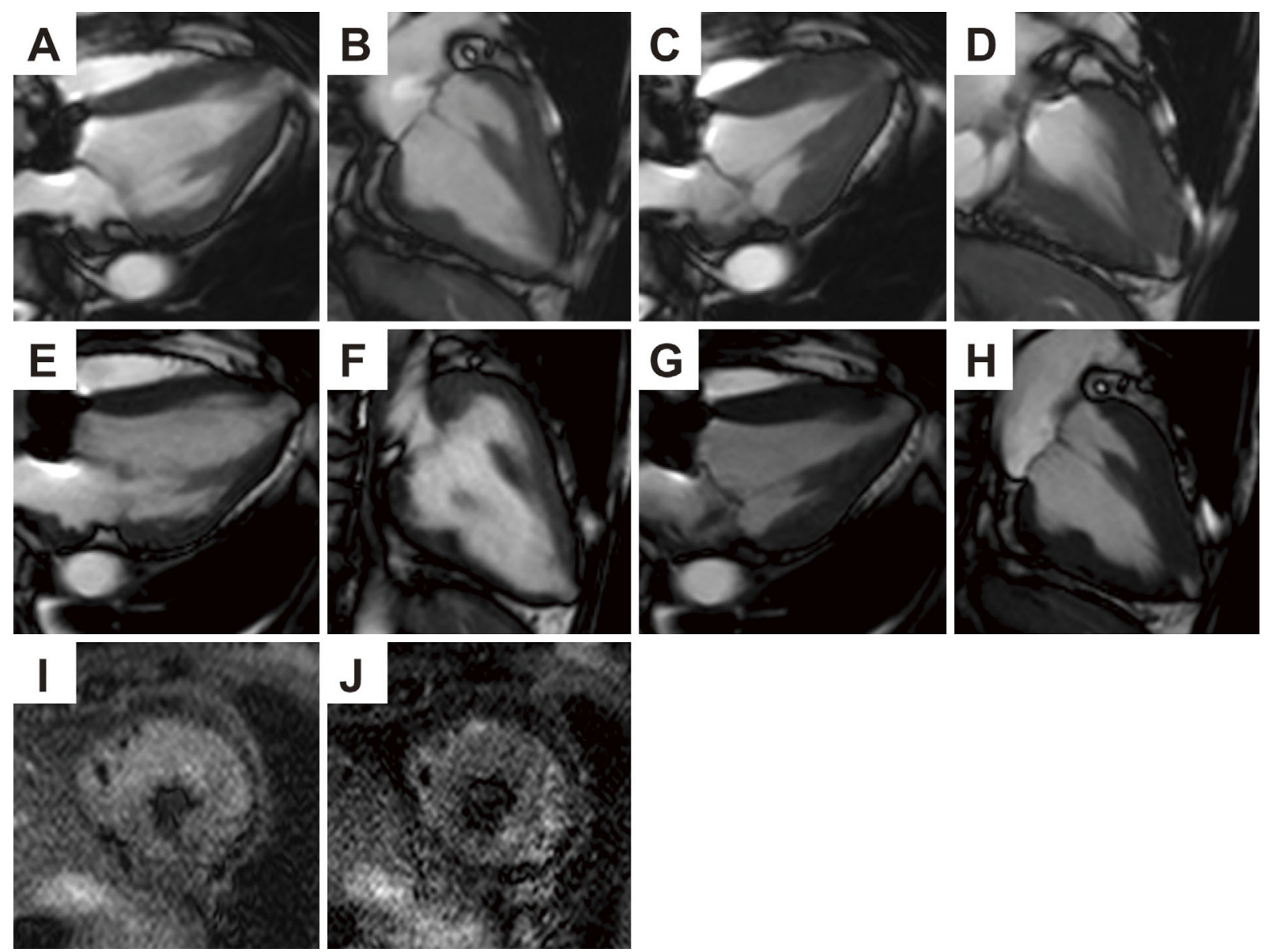

Figure 3. Cardiac MRI images obtained from case 1 at one and four months after the onset of takotsubo cardiomyopathy. At one month, LVWT and a left ventricular aneurysm were observed in end diastolic (A: long axis view, B: sagittal view) and systolic (C: long axis view, D: sagittal view) cine frames, while T2-weighted signal intensity at the apex increased (I: short axis view). At four months, the LVWT had recovered, but the left ventricular aneurysm persisted in the end diastolic (E: long axis view, F: sagittal view) and systolic (G: long axis view, H: sagittal view) cine frames, while T2weighted signal intensity improved (J: short axis view). MRI: magnetic resonance imaging, LVWT: left ventricular wall thickening 


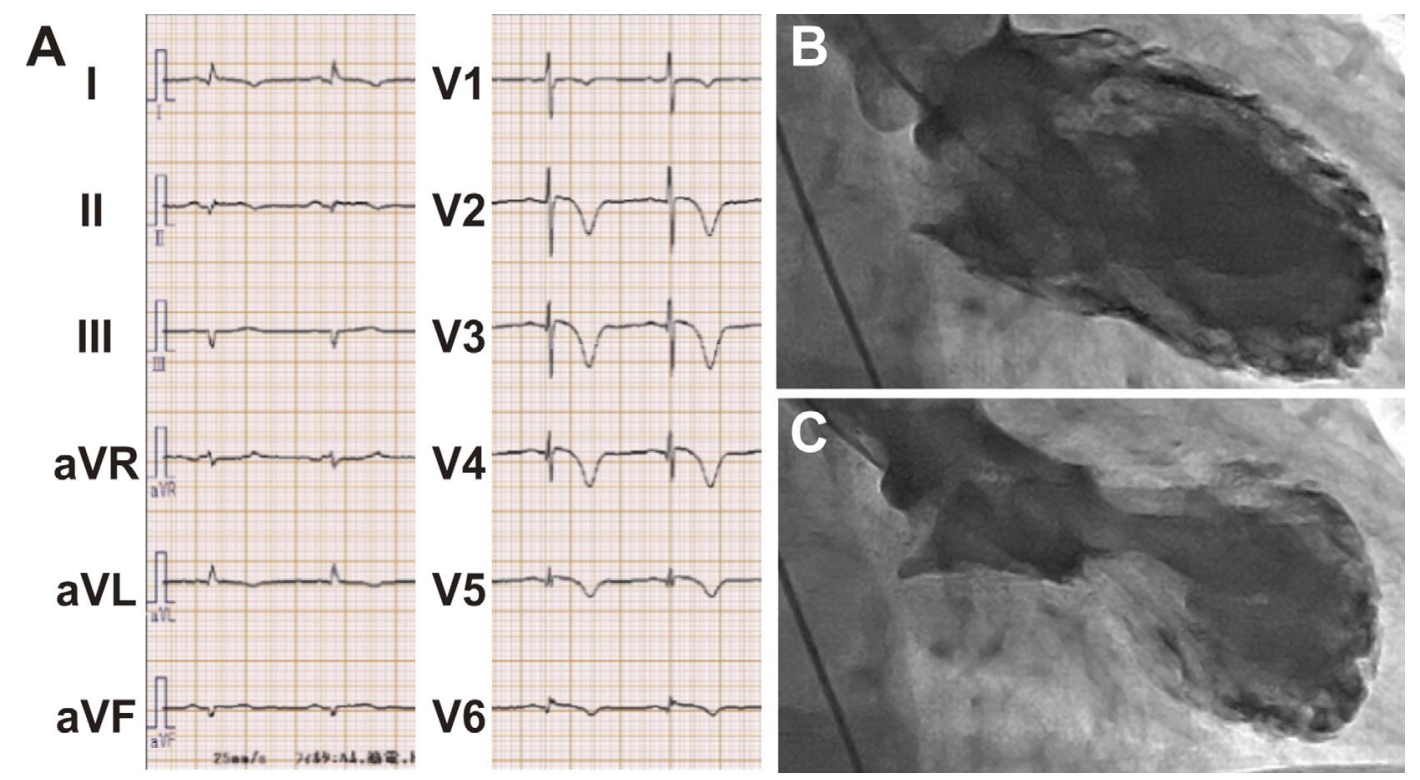

Figure 4. Left ventriculography and electrocardiography from case 2 at the onset of takotsubo syndrome. Her electrocardiogram showed $T$ wave inversion in multiple leads (A). Apical wall motion abnormality was detected by left ventriculography in end-diastole (B) and end-systole (C).
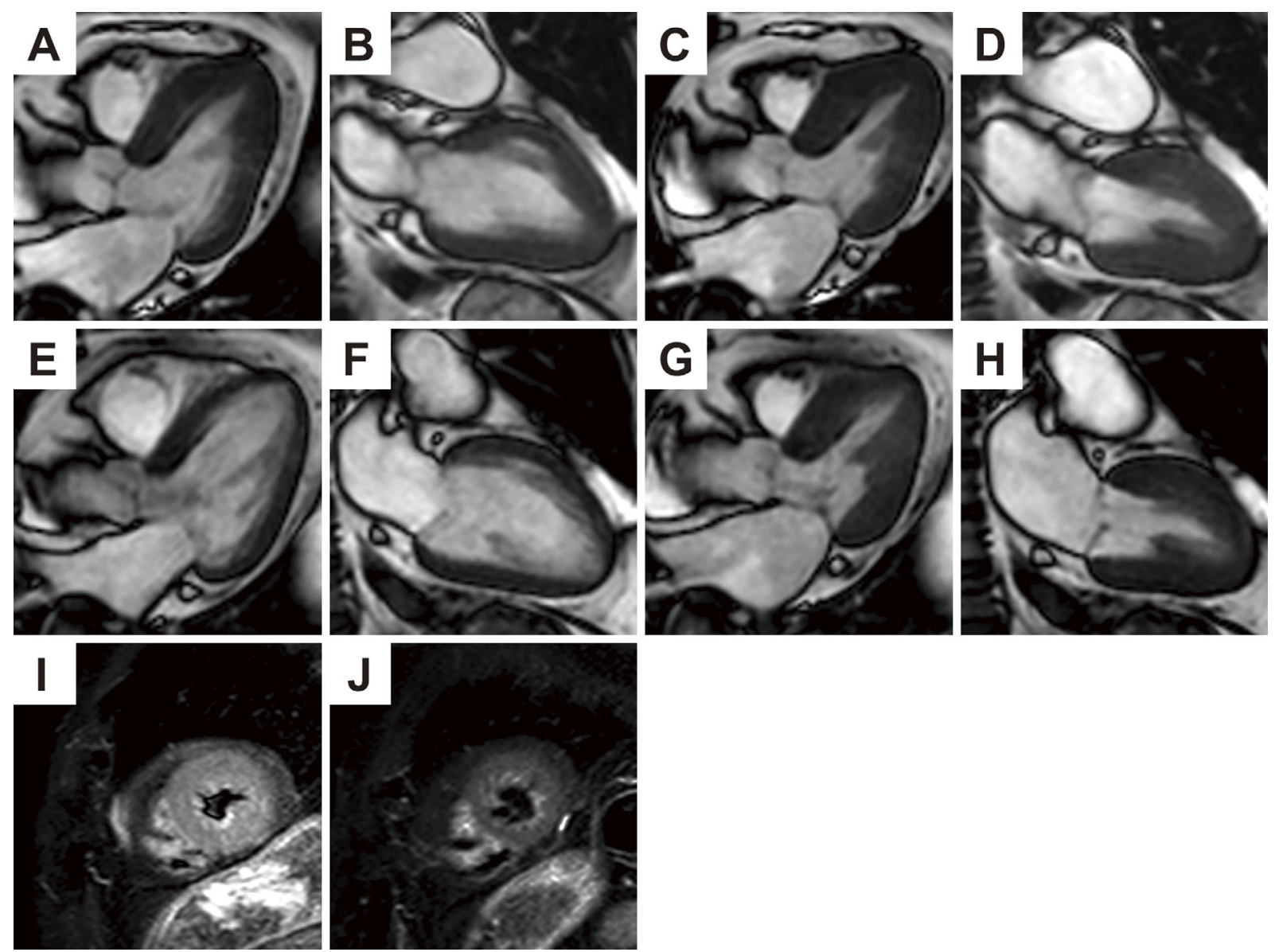

Figure 5. Cardiac MRI images obtained from case 2 at one and four months after the onset of takotsubo syndrome. At one month, LVWT was first observed in end diastolic (A: long axis view, B: sagittal view) and systolic (C: long axis view, D: sagittal view) cine frames, while the T2-weighted signal intensity at the apex increased (I: short axis view). At four months, the LVWT had recovered in end diastolic (E: long axis view, F: sagittal view) and systolic (G: long axis view, H: sagittal view) cine frames, while T2-weighted signal intensity improved (J: short axis view). MRI: magnetic resonance imaging, LVWT: left ventricular wall thickening 


\section{Discussion}

\section{Time course of LVWT}

In our two cases, LVWT was not observed in the acute phase, but it appeared after LV functional recovery. In a previous report, the initiation of LVWT was $14.6 \pm 10.3$ days after the initial diagnosis of takotsubo syndrome (3), but the duration was not clearly indicated. In case 1, LVWT was first detected on day 18 (days after the onset of takotsubo syndrome) and had not been noted on day 9. Furthermore, LVWT was still detectable by day 61 using echocardiography and was diminished by day 130, as confirmed by both echocardiography and cardiac MRI. In case two, LVWT was not observed on day 13 and was only first detected on day 20. The patient recovered slightly on day 104 , as indicated by echocardiography, and LVWT was undetectable by day 139, as indicated using cardiac MRI. In summary, LVWT developed around two to three weeks after the onset of takotsubo syndrome and persisted for about two to four months.

\section{Significance of T2 imaging and possible mechanism of LVWT}

Cardiac MRI is useful for evaluating myocardial tissue characterization because of its high spatial resolution and non-invasiveness. In this report, sequential cardiac MRI was obtained from two takotsubo syndrome patients with LVWT. Cardiac MRI showed high intensity on T2-weighted imaging that coincided with LVWT, indicating myocardial edema, as LVWT is a transient and reversible change (5). In a large multi-center study (6), a high signal intensity on T2weighted imaging was observed in $81 \%$ of patients with takotsubo syndrome, matching the distribution of LV dysfunction, although there were few reports of ventricular wall thickening. We considered the high signal intensity on T2weighted imaging to reflect myocardial edema and deemed it a necessary but not sufficient condition for LVWT.

We tried to identify the specific characteristics of takotsubo syndrome among patients with LVWT, but the severity of left ventricular functional disruptions and peak troponin I values at the time of onset of takotsubo syndrome were not markedly different in our two cases from those of a previous large cohort study (7). We were unable to obtain histological samples from our patients, but an endomyocardial biopsy might help clarify the pathophysiology of LVWT.

\section{Relationship between aneurysm and LVWT}

In case 1, a left ventricular apical aneurysm was detected using echocardiography and cardiac MRI. There are no other reports of left ventricular aneurysms accompanied by LVWT. However, ventricular aneurysms associated with takotsubo syndrome are not rare. The precise mechanism underlying left ventricular aneurysmal formation with takotsubo syndrome is still unknown, but the use of $\beta$-blockers was reported to be useful for its prevention (8), implying that ventricular wall stress due to a pressure gradient may be the cause of the aneurysm. These aneurysms are often large and life-threatening due to cardiac rupture. Many cases of cardiac rupture are reported to occur in the acute phase (usually within one week) with prolonged ST segment elevation (8). In case 1 , there were no aneurysms detected in the acute phase, so our case seemed to be different from previous cases in terms of the size and time course.

Cine MRI in case 1 clearly showed a diminished ventricular space near the apex at end-systole due to the thickened ventricular wall, while the ventricular cavity was retained in case 2. An apical aneurysm is known to occur with midventricular obstruction as a form of hypertrophic cardiomyopathy (9). The possible mechanism underlying apical aneurysm development in patients with hypertrophic cardiomyopathy is a result of apical infarction and elevated left ventricular apical pressures due to mid-ventricular obstruction (10). In our case, cardiac MRI revealed severe midventricular-to-apex wall-thickening, which is similar to hypertrophic cardiomyopathy on cine imaging, and apical LGE, suggesting the existence of an apico-basal pressure gradient and local necrosis that is frequently followed by an apical aneurysm. Indeed, the size of the aneurysm did not change after the recovery of wall thickening, which indicated that the main cause of aneurysmal formation was an apical pressure overload due to ventricular obstruction (Fig. 3). Although we did not attempt to measure the intraventricular pressure gradient by echocardiography in case 1 , the accelerated blood flow was difficult to detect because the narrowing of the ventricular cavity due to wall thickening was very close to the apex.

In addition, left ventricular aneurysm was also detected on echocardiography in our cases, but the poor visibility inherent in this technique is sometimes a problem, particularly in the apical segment. Therefore, cardiac MRI might be a more useful tool for detecting "hidden" ventricular aneurysms than echocardiography. Apical aneurysms often occur in association with thrombosis and arrhythmic events, such as ventricular tachycardia (11). Unfortunately, as our patient died due to sepsis seven months after the onset of takotsubo syndrome, we did not observe these complications. However aneurysmal development may have a role as a potential mechanism underlying the development of cerebral and cardiac complications accompanied by takotsubo syndrome.

\section{Conclusions}

This is the first case report of left ventricular aneurysm following transient LVWT with takotsubo syndrome. Although the precise mechanism of LVWT is still unknown, sequential cardiac MRI was helpful in detecting LVWT and performing follow-up to monitor its progress and detect complications.

\section{The authors state that they have no Conflict of Interest (COI).}




\section{References}

1. Kato T, Ban Y, Kuruma S, et al. Two cases of reversible left ventricular hypertrophy during recovery from takotsubo cardiomyopathy. Echocardiography 30: E92-E94, 2013.

2. Roy RR, Hakim FA, Hurst RT, Simper D, Appleton CP. Two cases of apical ballooning syndrome masking apical hypertrophic cardiomyopathy. Tex Heart Inst J 41: 179-183, 2014.

3. Shin DG, Cho IJ, Shim CY, et al. Transient apical wall thickening in patients with stress cardiomyopathy: Prevalence, profile, and impact on clinical course. Int J Cardiol 194: 87-92, 2015.

4. Friedrich MG, Sechtem U, Schulz-Menger J, et al. Cardiovascular magnetic resonance in myocarditis: a JACC white paper. J Am Coll Cardiol 53: 1475-1487, 2009.

5. Izgi C, Ray S, Nyktari E, et al. Myocardial edema in Takotsubo syndrome mimicking apical hypertrophic cardiomyopathy: an insight into diagnosis by cardiovascular magnetic resonance. Heart Lung 44: 481-485, 2015.

6. Eitel I, von Knobelsdorff-Brenkenhoff F, Bernhardt P, et al. Clinical characteristics and cardiovascular magnetic resonance findings in stress (takotsubo) cardiomyopathy. JAMA 306: 277-286, 2011.
7. Templin C, Ghadri JR, Diekmann J, et al. Clinical features and outcomes of takotsubo (stress) cardiomyopathy. N Engl J Med 373: 929-938, 2015.

8. Kumar S, Kaushik S, Nautiyal A, et al. Cardiac rupture in takotsubo cardiomyopathy: a systematic review. Clin Cardiol 34: 672676, 2011.

9. Webb JG, Sasson Z, Rakowski H, Liu P, Wigle ED. Apical hypertrophic cardiomyopathy: clinical follow-up and diagnostic correlates. J Am Coll Cardiol 15: 83-90, 1990.

10. Sivanandam A, Ananthasubramaniam K. Midventricular hypertrophic cardiomyopathy with apical aneurysm: potential for underdiagnosis and value of multimodality imaging. Case Rep Cardiol 2016: 9717948, 2016.

11. Lazaros G, Kouvousis N, Kotsanis A, Matsakas E. Apical hypertrophic cardiomyopathy with midventricular obstruction and apical aneurysm. Int J Cardiol 114: E45-E47, 2007.

The Internal Medicine is an Open Access article distributed under the Creative Commons Attribution-NonCommercial-NoDerivatives 4.0 International License. To view the details of this license, please visit (https://creativecommons.org/licenses/ by-nc-nd/4.0/).

(C) 2018 The Japanese Society of Internal Medicine Intern Med 57: 517-522, 2018 